\title{
Relação entre o índice inflamatório da dieta e o câncer de mama
}

Relationship between the inflammatory index in the diet and breast cancer

Relación entre el índice inflamatorio en la dieta y el cáncer de mama

Jardel Alves da Costa ORCID: https://orcid.org/0000-0002-9844-0770 Universidade Federal do Piauí, Brasil

E-mail: jardelalves@ufpi.edu.br

Iara Pereira Melo Moreira ORCID: https://orcid.org/0000-0003-3077-7577

Centro Universitário Santo Agostinho, Brasil

E-mail: iaramelo10@hotmail.com

Gabrielly Martins de Barros

ORCID: https://orcid.org/0000-0001-9696-424X

Universidade Federal do Piauí, Brasil

E-mail: gabyrhcp00@hotmail.com

Dênaba Luyla Lago Damasceno

ORCID: https://orcid.org/0000-0002-4608-4129

Centro Universitário Unifacid, Brasil

E-mail:denaba-luyla@hotmail.com

Renata Rodrigues Costa Fontinele

ORCID: https://orcid.org/0000-0002-6121-0322

Universidade Federal do Piaú, Brasil

E-mail: renatafontinele25@hotmail.com

Nayara Ferreira Ricardo

ORCID: https://orcid.org/0000-0002-6026-9728

Centro Universitário Maurício de Nassau, Brasil

E-mail:nayaratexumo@gmail.com

Juliane Barroso Leal

ORCID: https://orcid.org/0000-0001-7255-5732

Universidade Federal do Vale do São Francisco, Brasil

E-mail:juh_barroso@yahoo.com.br

Diêgo de Oliveira Lima

ORCID: https://orcid.org/0000-0001-8211-9416 Universidade Federal do Piauí, Brasil

E-mail: diego.oliveira@ufpi.edu.br

Fernanda Lopes Souza

ORCID: https://orcid.org/0000-0001-8280-9580 Universidade Federal do Piauí, Brasil

E-mail: fernandalopess0102@gmail.com

Monique Maria da Silva

ORCID: https://orcid.org/0000-0003-3554-5118 Universidade Federal do Piaú, Brasil

E-mail: monique.silva1201@ hotmail.com

Maria Janiele de Morais Silva

ORCID: https://orcid.org/0000-0002-5647-4315

Universidade Federal do Piauí, Brasil

E-mail: janiellymorais559@gmail.com

Whellyda Katrynne Silva Oliveira

ORCID: https://orcid.org/0000-0003-1920-1454

Universidade Federal do Piauí, Brasil

E-mail: oliveirawks@gmail.com

Ronnyely Suerda Cunha Silva

ORCID: https://orcid.org/0000-0003-3710-7824

Universidade Federal do Piauí, Brasi

E-mail: ronnyelynutricionista@gmail.com

Márcia Luiza dos Santos Beserra Pessoa

ORCID: https://orcid.org/0000-0001-8585-4264

Universidade Federal do Piauí, Brasil

E-mail: beserranut@hotmail.com 


\begin{abstract}
Resumo
Esta pesquisa tem como objetivo reunir estudos que evidenciem a relação entre o índice inflamatório da dieta (DII) e a incidência de câncer de mama $(\mathrm{CM})$. Trata-se de revisão integrativa da literatura. A seleção dos estudos foi realizada durante os meses de julho e agosto de 2021, nas bases de dados: LILACS, PUBMED e SCIENCE DIRECT via portal Periódico Capes. Inicialmente, a estratégia para a busca dos estudos foi composta pela combinação de descritores controlados (Índice; Inflamação; Dietas; Câncer de mama). Realizou-se a combinação dos descritores por intermédio do operador booleano AND. Após o processo de busca e seleção utilizando os critérios citados na metodologia, foram selecionados 5 artigos. Foi observado nos resultados dos estudos selecionados uma associação forte e independente entre o escore DII e a incidência de CM, no qual uma dieta de alto score DII se correlacionou a um risco aumentado da incidência de CM e o contrário também foi observado. Estudos futuros são necessários para obter informações sobre a relação entre a inflamação associada à dieta e o risco de CM. Estes achados aprofundam a compreensão sobre o papel da dieta na carcinogênese da mama.
\end{abstract}

Palavras-chave: Índice; Inflamação; Dietas; Câncer de mama.

\begin{abstract}
This research aims to bring together studies that show the relationship between the inflammatory index of the diet (IBD) and the incidence of breast cancer (CM). This is an integrative literature review. The selection of studies was carried out during the months of July and August 2021, in the databases: LILACS, PUBMED and SCIENCE DIRECT via Capes Periodical portal. Initially, the strategy for the search for studies was composed of a combination of controlled descriptors (Index; Inflammation; Diet; Breast cancer). The combination of descriptors was performed using the Boolean operator AND. After the search and selection process using the criteria mentioned in the methodology, 5 articles were selected. A strong and independent association between the DII score and the incidence of MC was observed in the results of the selected studies, in which a diet with a high DII score was correlated with an increased risk of the incidence of MC and the opposite was also observed. Future studies are needed to obtain information on the relationship between diet-associated inflammation and the risk of MC. These findings deepen the understanding of the role of diet in breast carcinogenesis.
\end{abstract}

Keywords: Index; Inflammation; Diets; Breast cancer.

\title{
Resumen
}

Esta investigación tiene como objetivo reunir estudios que muestren la relación entre el índice inflamatorio de la dieta (EII) y la incidencia de cáncer de mama (MC). Ésta es una revisión integradora de la literatura. La selección de estudios se realizó durante los meses de julio y agosto de 2021, en las bases de datos: LILACS, PUBMED y SCIENCE DIRECT a través del portal Capes Periodical. Inicialmente, la estrategia para la búsqueda de estudios estuvo compuesta por una combinación de descriptores controlados (Índice; Inflamación; Dieta; Cáncer de mama). La combinación de descriptores se realizó mediante el operador booleano AND. Tras el proceso de búsqueda y selección utilizando los criterios mencionados en la metodología, se seleccionaron 5 artículos. Se observó una asociación fuerte e independiente entre el puntaje DII y la incidencia de CM en los resultados de los estudios seleccionados, en los que una dieta con un puntaje alto de DII se correlacionó con un mayor riesgo de incidencia de CM y también se observó lo contrario. Se necesitan estudios futuros para obtener información sobre la relación entre la inflamación asociada a la dieta y el riesgo de CM. Estos hallazgos profundizan la comprensión del papel de la dieta en la carcinogénesis mamaria.

Palabras clave: Índice; Inflamación; Dietas; Cáncer de mama.

\section{Introdução}

O câncer de mama $(\mathrm{CM})$ é o câncer mais comum e uma das principais causas de mortalidade por câncer em mulheres em todo o mundo. Com base nas estimativas do GLOBOCAN de 2018, aproximadamente 2,1 milhões de novos casos de CM e 0,6 milhões de mortes por CM ocorreram em todo o mundo. O número de novos pacientes com este tipo de câncer aumentou aproximadamente 1,5 vezes em 2018, em comparação com 1,4 milhão de novos casos diagnosticados em 2008 (Ferlay et al., 2019; Jemal et al., 2011).

No Brasil, o câncer de mama é também o tipo de câncer mais incidente em mulheres de todas as regiões, após o câncer de pele não melanoma. As taxas são mais elevadas nas regiões mais desenvolvidas (Sul e Sudeste) e a menor é observada na região Norte. Em 2021, estima-se que ocorrerão 66.280 casos novos da doença, o que equivale a uma taxa de incidência de 43,74 casos por 100.000 mulheres (INCA, 2020). A incidência do câncer de mama tende a crescer progressivamente a partir dos 40 anos, assim como a mortalidade por essa neoplasia (INCA, 2019). 
A neoplasia mamária é mediada por hormônios como os esteroides (estrógeno e progesterona), prolactina (PRL), hormônio de crescimento (GH), fatores de crescimento (fator de crescimento tumoral - TGF e fator de crescimento epidermal EGF), e acentuada pelo efeito de fatores de risco sobre o estado hormonal e/ou alteração nos biomarcadores desse estado. Muitos desses fatores de risco não são modificáveis, como sexo, idade, raça, mutação genética e história familiar de CM (SOFI et al., 2018). Outros fatores de risco são potencialmente "modificáveis", tais como: atividade física, peso corporal, consumo de álcool e hábitos alimentares (Daly et al., 2021).

Apesar das inconsistências encontradas nos estudos, um padrão alimentar de alta qualidade foi sugerido como forma para reduzir o risco de CM (Norat et al., 2014). Uma dieta saudável, como o padrão dietético mediterrâneo e outros padrões dietéticos ricos em vegetais, frutas e peixes, são mencionados por exercer efeitos benéficos na prevenção do câncer por meio de diferentes mecanismos envolvidos na iniciação, promoção e progressão do câncer (Albuquerque, Baltar \& Marchioni, 2014, Gardeazabal et al., 2019). Alguns dos mecanismos relatados são: efeito antioxidante (devido à grande quantidade de polifenóis nesses alimentos), inibição do crescimento e potencial metastático de células cancerosas, inibição da autofagia, repressão transcricional do receptor do fator de crescimento epidérmico humano 2 (HER2), inibição da proliferação celular e invasividade, inibição da aromatase e redução da produção de estrogênio endógeno. Evidências epidemiológicas indicam que a dieta também influencia fortemente a inflamação crônica, um dos principais fatores da fisiopatologia do câncer (Yu et al., 2016).

A inflamação crônica é um importante contribuinte para o desenvolvimento e progressão dos cânceres, incluindo o CM. A inflamação crônica pode causar danos ao DNA e estimular a proliferação celular crônica, que são fatores importantes na carcinogênese. Um grande corpo de evidências indica que biomarcadores inflamatórios circulantes, incluindo interleucina (IL) -4, IL-6, IL-8, proteína C reativa (CRP) e fator de necrose tumoral- $\alpha$ (TNF- $\alpha$ ), podem estar associados com risco de câncer de mama. (Knüpfer \& Preiß, 2007; Onuchic \& Chammas, 2010; Fernandes et al., 2020).

Recentemente, estudos sugeriram achados contraditórios a respeito da relação entre a sobrevida após o diagnóstico de câncer de mama e nutrientes específicos conhecidos por modular a inflamação, como gordura dietética, frutas, vegetais, fibras e álcool. O potencial inflamatório geral da dieta pode fornecer melhores percepções sobre o efeito da dieta na sobrevivência ao câncer de mama do que avaliar apenas um único nutriente, a final, uma dieta humana típica consiste em uma variedade de alimentos e nutrientes pró-inflamatórios e antiinflamatórios (Chlebowski et al., 2006; Chlebowski et al., 2017; Zheng et al., 2018).

O Índice Inflamatório Dietético (DII) foi criado para investigar de forma abrangente o potencial inflamatório da dieta na população em geral com ajuste para diferenças dietéticas em todo o mundo. Este índice foi desenvolvido por meio de uma extensa revisão de mais de 6.500 artigos publicados de 1950 a 2010. Quarenta e cinco parâmetros alimentares (incluindo vários macronutrientes, micronutrientes, flavonóides e itens alimentares individuais) foram avaliados, e seis biomarcadores inflamatórios foram usados para calcular as características inflamatórias de cada parâmetro alimentar (Shivappa et al., 2014; Vasconcelos Filho et al., 2020).

Um parâmetro alimentar é considerado pró-inflamatório se sua ingestão estiver associada a aumentos significativos nos biomarcadores inflamatórios, ou anti-inflamatório e se estiver associada a diminuições significativas de biomarcadores inflamatórios. Uma pontuação é atribuída a cada parâmetro alimentar com base em sua associação com biomarcadores inflamatórios na literatura, e a pontuação é ponderada com base no desenho do estudo. As pontuações são então ajustadas à ingestão média padrão global, convertidas em proporções (0-1) e distribuídas em torno de zero, com pontuações DII mais altas indicando efeitos pró-inflamatórios e pontuações DII mais baixas sugerindo efeitos anti-inflamatórios (Chen et al., 2021).

É relevante considerar que os alimentos frequentemente consumidos, com seus nutrientes e compostos bioativos, estabelecem uma relação com marcadores de inflamação, atribuindo à dieta habitual uma característica pró ou anti- 
inflamatória. Escores elevados de DII, correspondendo a dietas mais pró-inflamatórias, foram positivamente associados ao risco de câncer de mama em estudos clínicos recentes (Huang et al., 2017; Jalali et al., 2018).

Diante do contexto relatado, esta pesquisa tem como objetivo reunir estudos que evidenciem a relação entre o índice inflamatório da dieta e a incidência de câncer de mama.

\section{Metodologia}

Trata-se de revisão integrativa da literatura, conduzida conforme orientações de Souza, Silva e Carvalho (2010). Assim, o estudo seguiu as etapas: elaboração da pergunta norteadora, busca ou amostragem na literatura, coleta de dados, análise crítica dos estudos incluídos, discussão dos resultados e apresentação da revisão integrativa (Souza, silva \& carvalho, 2010).

A seleção dos estudos foi realizada durante os meses de julho e agosto de 2021, nas bases de dados: Literatura LatinoAmericana e do Caribe em Ciências da Saúde (LILACS), National Library of Medicine (PUBMED) e Biblioteca virtual da Elsevier (SCIENCE DIRECT) via portal Periódico Capes.

Inicialmente, a estratégia para a busca dos estudos foi composta pela combinação de descritores controlados (Índice; Inflamação; Dietas; Câncer de mama) indexados nos Descritores em Ciências da Saúde (DeCS/MeSH). Realizou-se a combinação dos descritores controlados por intermédio do operador booleano AND como demonstrado na Tabela 1.

Tabela 1 - Estratégias de busca utilizadas nas bases de LILACS, PUBMED e SCIENCE DIRECT.

\begin{tabular}{|c|c|c|c|c|}
\hline $\begin{array}{c}\text { BASE DE } \\
\text { DADOS }\end{array}$ & ESTRATÉGIA DE BUSCA & RESULTADOS & FILTRADOS & SELECIONADOS \\
\hline Lilacs & $\begin{array}{l}\text { "Indice and Inflamação and } \\
\text { Dietas and Cancer de mama" } \\
\text { "Index and Inflammation and } \\
\text { Diets and Breast Cancer" }\end{array}$ & 0 & 0 & 0 \\
\hline PubMed & $\begin{array}{l}\text { "Indice and Inflamação and } \\
\text { Dietas and Cancer de mama" } \\
\text { "Index and Inflammation and } \\
\text { Diets and Breast Cancer" }\end{array}$ & 17941 & 250 & 4 \\
\hline Science Direct & $\begin{array}{l}\text { "Indice and Inflamação and } \\
\text { Dietas and Cancer de mama" } \\
\text { "Index and Inflammation and } \\
\text { Diets and Breast Cancer" }\end{array}$ & 9794 & 52 & 1 \\
\hline
\end{tabular}

Fonte: Pesquisa direta (2021).

Além da utilização dos descritores, a seleção dos estudos ocorreu de acordo com os critérios de inclusão que foram: artigos originais completos que realizaram avaliação do impacto do DII na incidência do CM. Trabalhos publicados entre os anos de 2016 e 2021 nos idiomas inglês e português, com a presença dos referidos descritores e trabalhos gratuitos disponíveis na integra. Foram excluídos artigos duplicados, incompletos e estudos realizados com animais.

O levantamento nas bases de dados resultou em 12 artigos em português e 27.723 artigos em inglês. Algumas referências foram utilizadas após busca manual dos artigos selecionados. A triagem da busca revelou 15 estudos duplicados, resultando em 27.708 registros após remoção das duplicações.

$\mathrm{Na}$ primeira triagem, baseada nos critérios de inclusão e exclusão, eliminaram-se 27.550 estudos. Após a elegibilidade/avaliação crítica dos textos completos de 158 registros, 5 atenderam aos critérios de inclusão e prosseguiram para 
fase de extração de dados, leitura exaustiva e síntese do conhecimento. A Figura 1 representa o fluxograma do processo de busca e seleção detalhados.

Figura 1 - Fluxograma do processo de busca e seleção de estudos.

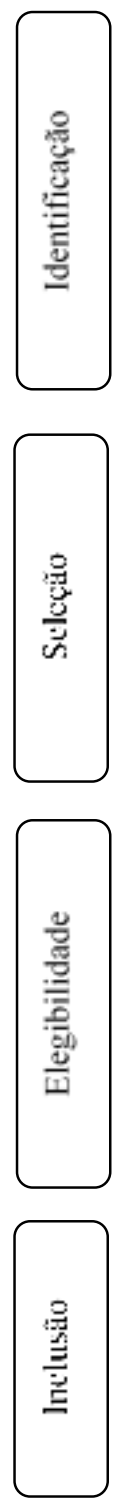

Estudos identificados:

Liluts: $(0$ - português; 0 - inglês 0 .

PubMed : (12-português; 17929 -ing̣lt̂́s 1794l).

Science Direct: 0 -português; 2363 - ing̣lîs 2363 ). resumos:

Lilucs: $(0$ - português; 0 - inglı̂s 0$)$.

PubMed : ( 0 - português; 250 - ing̣lîs 250$)$.

Science Direct: 10 - português; 52 - in니농 52 ).
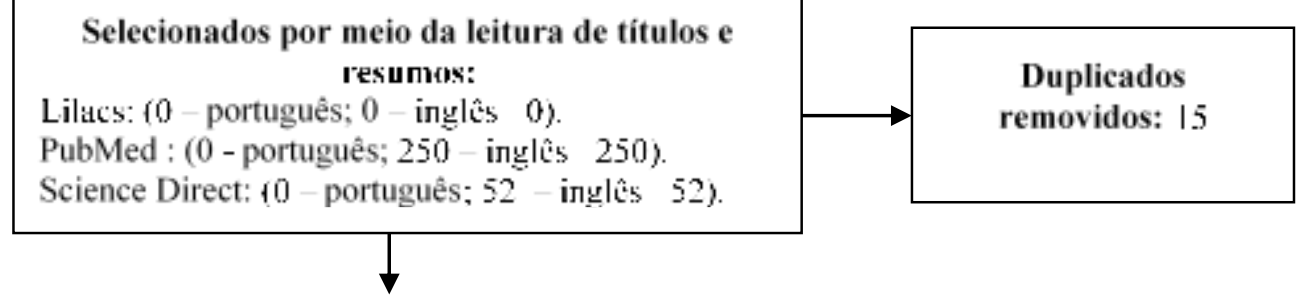

Excluídos após a leitura de títulos e resumos:

Lilacs: $(0$ português; 0 inğlês $=0)$.

PubMed : $(0$ - português; 2.35 ingllês $=2.35)$.

Science Direct: $(0$ - português; 52 inğlês $=52)$.
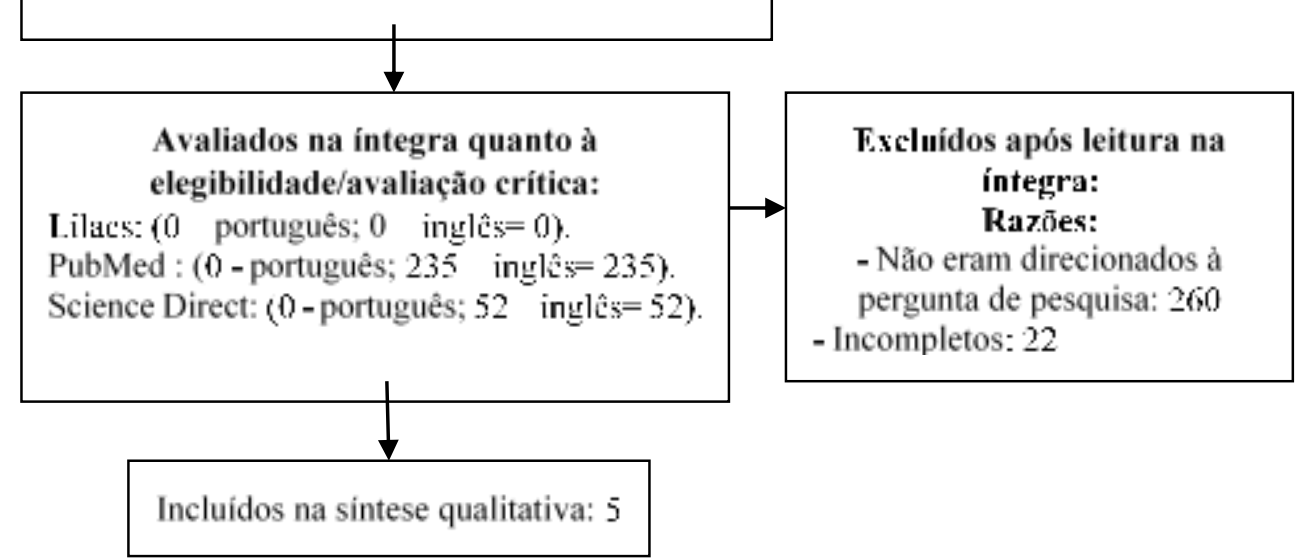

Fonte: Adaptado de Neely, Adams e Crowe (2001).

\section{Resultados e Discussão}

Após o processo de busca e seleção utilizando os critérios citados na metodologia, foram selecionados 5 artigos. Na Tabela 2 é possível visualizar os autores e ano de publicação, tipo de estudo, metodologia e principais resultados relatados por cada estudo. 
Tabe1a 2 - Descrição dos estudos selecionados.

\begin{tabular}{|c|c|c|}
\hline Autores/ano & Tipo de estudo/ Metodologia & Resultados \\
\hline Shivappa et al. (2017). & $\begin{array}{l}\text { Estudo de caso-controle/Participaram do } \\
\text { estudo } 2.569 \text { mulheres com CM. Os escores } \\
\text { DII foram calculados a partir da ingestão } \\
\text { alimentar avaliada por um questionário de } \\
\text { frequência alimentar. }\end{array}$ & $\begin{array}{l}\text { O alto escore DII foi significativamente associado a um risco } \\
\text { aumentado de câncer de mama de maneira que a relação de DII } \\
\text { com a incidência de CM é independente dos efeitos de outros } \\
\text { fatores de risco, como idade, IMC, estado da menopausa, } \\
\text { paridade, história familiar de cânceres sensíveis a hormônios e } \\
\text { ingestão de energia. }\end{array}$ \\
\hline Jang et al. (2018). & $\begin{array}{l}\text { Estudo de coorte/ Participaram do estudo } \\
511 \text { mulheres com CM. Os escores DII } \\
\text { foram calculados a partir da ingestão } \\
\text { alimentar avaliada por um questionário de } \\
\text { frequência alimentar. }\end{array}$ & $\begin{array}{l}\text { O alto escore DII foi significativamente maior em pacientes com } \\
\text { recorrência de CM do que aqueles sem recorrência. As taxas de } \\
\text { sobrevida livre de doença e sobrevida global foram } \\
\text { significativamente menores em pacientes com escores DII mais } \\
\text { elevados. Além disso, o DII foi positivamente associado ao risco } \\
\text { de recorrência do câncer de acordo com fatores, como idade } \\
\text { (menor que }<50 \text { anos), estado pré-menopausa, índice de massa } \\
\text { corporal (maior que ou igual } \geq 25 \mathrm{~kg} / \mathrm{m} \text { ). }\end{array}$ \\
\hline Vahid et al. (2018). & $\begin{array}{l}\text { Estudo de caso-controle/Participaram do } \\
\text { estudo } 145 \text { pacientes com CM. Os escores } \\
\text { DII foram calculados a partir da ingestão } \\
\text { alimentar avaliada por um questionário de } \\
\text { frequência alimentar. }\end{array}$ & $\begin{array}{l}\text { O alto escore DII foi significativamente associado a risco } \\
\text { aumentado de CM enquanto scores mais baixos foram } \\
\text { positivamente associados a menor recorrência de CM. Esse } \\
\text { resultado apoiou a hipótese de que o consumo de uma dieta mais } \\
\text { pró-inflamatória é um fator de risco fisiopatológico para o CM. }\end{array}$ \\
\hline Lee et al. (2019). & $\begin{array}{l}\text { Estudo de caso-controle/Participaram do } \\
\text { estudo } 364 \text { pacientes com CM e } 364 \\
\text { controles pareados por idade. Os escores } \\
\text { DII foram calculados a partir da ingestão } \\
\text { alimentar avaliada por um questionário de } \\
\text { frequência alimentar. }\end{array}$ & $\begin{array}{l}\text { O escore DII foi significativamente maior nos casos do que nos } \\
\text { controles. Descobriu-se que pontuações DII mais altas foram } \\
\text { relacionadas a um risco aumentado de CM para receptor de } \\
\text { estrogênio e receptor de progesterona. }\end{array}$ \\
\hline Wang et al. (2020). & $\begin{array}{l}\text { Estudo de coorte/ Participaram do estudo } \\
1.064 \text { mulheres com CM. Os escores DII } \\
\text { foram calculados a partir da ingestão } \\
\text { alimentar avaliada por um questionário de } \\
\text { frequência alimentar. }\end{array}$ & $\begin{array}{l}\text { O baixo escore DII foi significativamente associado a riscos mais } \\
\text { baixos de mortalidade por causas diversas como caquexia e } \\
\text { mortalidade específica por CM. Esses riscos variaram de acordo } \\
\text { com o período de acompanhamento, os efeitos protetores do } \\
\text { consumo de dieta anti-inflamatória no prognóstico do CM } \\
\text { tornaram-se mais fortes nos casos de acompanhamento a longo } \\
\text { prazo. }\end{array}$ \\
\hline
\end{tabular}

Fonte: Dados da pesquisa (2021).

O estudo de Shivappa et al. (2017) avaliou a associação entre o DII e as chances de CM e concluiu que uma dieta próinflamatória (ou seja, de alto score DII) está associada ao aumento do risco de CM. Já em um estudo anterior de caso-controle conduzido na Alemanha, nenhuma associação significativa foi observada entre o escore DII e o risco de CM em mulheres (Ge et al., 2015).

O estudo de Jang et al. (2018) mostrou que dietas anti-inflamatórias podem diminuir o risco de recorrência do câncer e mortalidade geral em pacientes com câncer de mama, particularmente aquelas com fatores, como idade mais jovem, estado de pré-menopausa, obesidade, tamanho do tumor (maior que $>2 \mathrm{~cm}$ ) e presença de metástases em linfonodos. A pontuação DII foi menor e geralmente mais estreita nas mulheres italianas do que nas americanas porque as mulheres italianas aderiram mais a uma dieta mediterrânea, que está negativamente correlacionada com a pontuação DII.

Outros estudos mostraram que o DII foi positivamente associado à incidência de CM em mulheres chinesas, italianas, americanas e suecas (Shivappa et al., 2017; Shivappa et al., 2015), mas não em mulheres americanas e alemãs (Tabung et al., 2016; Ge et al., 2015). A inconsistência na associação entre o escore DII e o risco de incidência de CM não estão totalmente elucidadas (Jang et al., 2018). 
O estudo de Vahid et al. (2018) concluiu que as mulheres que consumiram uma dieta mais pró-inflamatória conforme indicado por escores DII mais elevados, tiveram maior risco de CM em comparação com as mulheres que consumiram uma dieta mais anti-inflamatória. Assim, encorajando a ingestão de mais nutrientes anti-inflamatórios, como ácidos graxos ômega3, alimentos vegetais ricos em fibras, beta-caroteno e outros carotenóides e fitoquímicos, e reduzindo a ingestão de alimentos pró-inflamatórios, como frituras ou alimentos processados, ricos em gordura saturada ou ácidos graxos trans, como uma estratégia para reduzir o risco da incidência de CM. Um dos possíveis mecanismos para a associação positiva entre o DII e o risco de $\mathrm{CM}$ e outros estados inflamatórios crônicos pode ser através do efeito de uma dieta pró-inflamatória na resistência à insulina, que é conhecida por aumentar a inflamação sistêmica (Festa et al., 2000; Vahid et al., 2016).

O estudo de Lee et al. (2019) demonstrou a associação entre o potencial inflamatório da dieta, medido pelos escores DII, e o risco de CM em mulheres coreanas. Foram utilizados 37 parâmetros dietéticos que incluíam alimentos antiinflamatórios, incluindo alho, cebola e pimenta, bem como componentes de nutrientes para calcular a pontuação DII. Foi relatado que altos escores DII foram associados positivamente com o risco de CM. Esses resultados apoiam a hipótese de que as mulheres que consomem uma dieta mais pró-inflamatória podem ter maior risco desenvolver este tipo de câncer.

Estudos anteriores avaliaram a relação entre nutrientes e certos itens alimentares e marcadores inflamatórios circulantes (Esmaillzadeh et al., 2006; Kitabchi et al., 2013; Ma et al., 2006). Os parâmetros nutricionais e alimentares incluídos no DII foram selecionados com base no efeito de cada parâmetro na anti-inflamação ou pró-inflamação. Assim, os componentes individuais do DII também estão ligados a mudanças nos marcadores de inflamação (Shivappa et al., 2014).

O estudo de Wang et al. (2020) examinou a associação entre índice inflamatório alimentar pós-diagnóstico (DII®) e riscos de morte por causas diversas e mortalidade específica por CM. Os autores concluíram que a dieta anti-inflamatória (ou seja, de baixo score DII) de longo prazo pode ser um meio de melhorar a sobrevida de sobreviventes do CM, pois esta foi negativamente associada ao risco de morte nos pacientes com CM.

Uma análise prospectiva da Women's Health Initiative realizada por Tabung et al. (2016) relatou que o potencial inflamatório alimentar antes do diagnóstico está relacionado à morte por $\mathrm{CM}$, no entanto, estudos futuros são necessários para examinar o potencial inflamatório da dieta pós-diagnóstico, que é uma abordagem importante para conduzir a intervenção dietética no processo de prevenção do CM secundário.

\section{Considerações Finais}

Em conclusão, foi observada nos resultados dos estudos selecionados uma associação forte e independente entre o escore DII, no qual uma dieta de alto score DII se correlacionou a um risco aumentado da incidência de CM e o contrário também foi observado.

Estudos futuros devem ser realizados para se obter informações sobre a relação entre a inflamação associada à dieta e o risco de $\mathrm{CM}$, isso aprofundaria a compreensão sobre o papel da dieta na carcinogênese da mama. Pesquisas futuras também são necessárias para avaliar se o potencial inflamatório da dieta pode reduzir a inflamação crônica e o risco de CM. Ao fazer isso, a utilidade do DII pode ser estendida a ambientes clínicos para lidar com a potência inflamatória da dieta dos pacientes e, possivelmente, reduzir o risco futuro de doenças inflamatórias crônicas como o CM.

\section{Referências}

Albuquerque, R. C., Baltar, V. T., \& Marchioni, D. M. (2014). Breast cancer and dietary patterns: a systematic review. Nutrition reviews, $72(1)$, 1-17.

Chen, H., Gao, Y., Wei, N., Du, K., \& Jia, Q. (2021). Strong association between the dietary inflammatory index (DII) and breast cancer: a systematic review and meta-analysis. Aging (Albany NY), 13(9), 13039. 
Chlebowski, R. T., Aragaki, A. K., Anderson, G. L., Thomson, C. A., Manson, J. E., Simon, M. S., \& Prentice, R. L. (2017). Low-fat dietary pattern and breast cancer mortality in the Women's Health Initiative randomized controlled trial. Journal of Clinical Oncology, 35(25), 2919.

Chlebowski, R. T., Blackburn, G. L., Thomson, C. A., Nixon, D. W., Shapiro, A., Hoy, M. K., \& Elashoff, R. M. (2006). Dietary fat reduction and breast cancer outcome: interim efficacy results from the Women's Intervention Nutrition Study. Journal of the National Cancer Institute, $98(24), 1767-1776$.

Daly, A. A., Rolph, R., Cutress, R. I., \& Copson, E. R. (2021). A Review of Modifiable Risk Factors in Young Women for the Prevention of Breast Cancer. Breast Cancer: Targets and Therapy, 13, 241.

Esmaillzadeh, A., Kimiagar, M., Mehrabi, Y., Azadbakht, L., Hu, F. B., \& Willett, W. C. (2006). Fruit and vegetable intakes, C-reactive protein, and the metabolic syndrome. The American journal of clinical nutrition, 84(6), 1489-1497.

Ferlay, J., Colombet, M., Soerjomataram, I., Mathers, C., Parkin, D. M., Piñeros, M., \& Bray, F. (2019). Estimating the global cancer incidence and mortality in 2018: GLOBOCAN sources and methods. International journal of cancer, 144(8), 1941-1953.

Fernandes, D. P., Coutinho, V. E. A., de Brito Medeiros, L., \& Pereira, N. L. V. (2020). Nutrientes e compostos bioativos na modulação epigenética associada à prevenção e combate ao câncer. Research, Society and Development, 9(4), e114942914-e114942914.

Festa, A., D’Agostino Jr, R., Howard, G., Mykkanen, L., Tracy, R. P., \& Haffner, S. M. (2000). Chronic subclinical inflammation as part of the insulin resistance syndrome: the Insulin Resistance Atherosclerosis Study (IRAS). Circulation, 102(1), 42-47.

Gardeazabal, I., Ruiz-Canela, M., Sánchez-Bayona, R., Romanos-Nanclares, A., Aramendía-Beitia, J. M., Shivappa, N., \& Toledo, E. (2019). Dietary inflammatory index and incidence of breast cancer in the SUN project. Clinical Nutrition, 38(5), 2259-2268.

Ge, I., Rudolph, A., Shivappa, N., Flesch-Janys, D., Hebert, J. R., \& Chang-Claude, J. (2015). Dietary inflammation potential and postmenopausal breast cancer risk in a German case-control study. The Breast, 24(4), 491-496.

Huang W. Q., Mo X. F., Y, Y. B. et al. A higher Dietary Inflammatory Index score is associated with a higher risk of breast cancer among Chinese women: A case-control study. British Journal of Nutrition, 2017; 117(10): 1358-1367

Jang, H., Chung, M. S., Kang, S. S., \& Park, Y. (2018). Association between the dietary inflammatory index and risk for cancer recurrence and mortality among patients with breast cancer. Nutrients, 10(8), 1095.

Jalali, S., Shivappa, N., Hébert, J. R., Heidari, Z., Hekmatdoost, A., \& Rashidkhani, B. (2018). Dietary inflammatory index and odds of breast cancer in a casecontrol study from Iran. Nutrition and cancer, 70(7), 1034-1042.

Jemal, A., Bray, F., Center, M. M., Ferlay, J., Ward, E., \& Forman, D. (2011). Global cancer statistics. CA: a cancer journal for clinicians, 61(2), 69-90. Jung, K. W., Won, Y. J., Kong, H. J., \& Lee, E. S. (2019). Prediction of cancer incidence and mortality in Korea, 2019. Cancer research and treatment: official journal of Korean Cancer Association, 51(2), 431.

Kitabchi, A. E., McDaniel, K. A., Wan, J. Y., Tylavsky, F. A., Jacovino, C. A., Sands, C. W., \& Stentz, F. B. (2013). Effects of high-protein versus highcarbohydrate diets on markers of $\beta$-cell function, oxidative stress, lipid peroxidation, proinflammatory cytokines, and adipokines in obese, premenopausal women without diabetes: a randomized controlled trial. Diabetes care, 36(7), 1919-1925.

Knüpfer, H., \& Preiß, R. (2007). Significance of interleukin-6 (IL-6) in breast cancer. Breast cancer research and treatment, 102(2), 129-135

Lee, S., Quiambao, A. L., Lee, J., Ro, J., Lee, E. S., Jung, S. Y., \& Kim, J. (2019). Dietary inflammatory index and risk of breast cancer based on hormone receptor status: a case-control study in Korea. Nutrients, 11(8), 1949.

Ma, Y., Griffith, J. A., Chasan-Taber, L., Olendzki, B. C., Jackson, E., Stanek III, E. J., \& Ockene, I. S. (2006). Association between dietary fiber and serum C-reactive protein. The American journal of clinical nutrition, 83(4), 760-766.

Neely, A., Adams, C., \& Crowe, P. (2001). The performance prism in practice. Measuring business excellence.

Norat, T., Aune, D., Chan, D., \& Romaguera, D. (2014). Fruits and vegetables: updating the epidemiologic evidence for the WCRF/AICR lifestyle recommendations for cancer prevention. Advances in nutrition and cancer, 35-50.

Onuchic, A. C., \& Chammas, R. (2010). Câncer e o microambiente tumoral. Revista de medicina, 89(1), 21-31.

Shivappa, N., Blair, C. K., Prizment, A. E., Jacobs, D. R., \& Hébert, J. R. (2017). Prospective study of the dietary inflammatory index and risk of breast cancer in postmenopausal women. Molecular nutrition \& food research, 61(5), 1600592.

Shivappa, N., Sandin, S., Löf, M., Hébert, J. R., Adami, H. O., \& Weiderpass, E. (2015). Prospective study of dietary inflammatory index and risk of breast cancer in Swedish women. British journal of cancer, 113(7), 1099-1103.

Shivappa, N., Steck, S. E., Hurley, T. G., Hussey, J. R., \& Hébert, J. R. (2014). Designing and developing a literature-derived, population-based dietary inflammatory index. Public health nutrition, 17(8), 1689-1696.

Souza, M. T. D., Silva, M. D. D., \& Carvalho, R. D. (2010). Revisão integrativa: o que é e como fazer. Einstein (São Paulo), 8, $102-106$.

Tabung, F. K., Steck, S. E., Liese, A. D., Zhang, J., Ma, Y., Caan, B., \& Hebert, J. R. (2016). Association between dietary inflammatory potential and breast cancer incidence and death: results from the Women's Health Initiative. British journal of cancer, 114(11), 1277-1285.

Vahid, F., Shivappa, N., Hatami, M., Sadeghi, M., Ameri, F., Naeini, Y. J., \& Davoodi, S. H. (2018). Association between dietary inflammatory index (DII) and risk of breast cancer: a case-control study. Asian Pacific journal of cancer prevention: APJCP, $19(5), 1215$. 
Research, Society and Development, v. 10, n. 13, e432101320488, 2021 (CC BY 4.0) | ISSN 2525-3409 | DOI: http://dx.doi.org/10.33448/rsd-v10i13.20488

Vahid, F., Shivappa, N., Karamati, M., Naeini, A. J., Hebert, J. R., \& Davoodi, S. H. (2016). Association between Dietary Inflammatory Index (DII) and risk of prediabetes: a case-control study. Applied Physiology, Nutrition, and Metabolism, 42(4), 399-404.

Vasconcelos Filho, W. C. P., Cacau, L. T., de Carvalho Sampaio, H. A., Carioca, A. A. F., Rocha, D. C., de Oliveira Farias, B., \& Arruda, S. P. M. (2020). Associação entre o fator inflamatório dietético com indicadores de obesidade em homens com câncer de próstata. Research, Society and Development, 9(9), e499997557-e499997557.

Wang, K., Sun, J. Z., Wu, Q. X., Li, Z. Y., Li, D. X., Xiong, Y. F., \& Ren, G. S. (2020). Long-term anti-inflammatory diet in relation to improved breast cancer prognosis: a prospective cohort study. NPJ breast cancer, 6(1), 1-11.

Yu, J., Bi, X., Yu, B., \& Chen, D. (2016). Isoflavones: anti-inflammatory benefit and possible caveats. Nutrients, 8(6), 361.

Zheng, J., Tabung, F. K., Zhang, J., Liese, A. D., Shivappa, N., Ockene, J. K., \& Steck, S. E. (2018). Association between post-cancer diagnosis dietary inflammatory potential and mortality among invasive breast cancer survivors in the Women's Health Initiative. Cancer Epidemiology and Prevention Biomarkers, 27(4), 454-463.

Instituto Nacional De Câncer José Alencar Gomes Da Silva. Estimativa 2020: incidência do Câncer no Brasil. INCA, 2019. https://www.inca.gov.br/sites/ufu.sti.inca.local/files//media/document//.

Lee, S., Quiambao, A. L., Lee, J., Ro, J., Lee, E. S., Jung, S. Y., \& Kim, J. (2019). Dietary inflammatory index and risk of breast cancer based on hormone receptor status: a case-control study in Korea. Nutrients, 11(8), 1949.

Chen, W., Mo'ez Al-Islam, EF, Bragazzi, NL, AlGahtani, HM, Saif, Z., Jahrami, A., \& Jahrami, H. (2021). A inflamação relacionada à dieta está associada ao transtorno depressivo maior em adultos do Bahrein: resultados de um estudo caso-controle usando o índice inflamatório alimentar. Journal of Inflammation Research, 14, 1437 